\title{
Time-resolved detection of surface plasmon polaritons with a scanning tunneling
} microscope

Keil, Ulrich Dieter Felix; Ha, T.; Jensen, Jacob Riis; Hvam, Jørn Märcher

Published in:

Summaries of papers presented at the Conference on Lasers and Electro-Optics

Link to article, DOI:

10.1109/CLEO.1998.676596

Publication date:

1998

Document Version

Publisher's PDF, also known as Version of record

Link back to DTU Orbit

Citation $(A P A)$ :

Keil, U. D. F., Ha, T., Jensen, J. R., \& Hvam, J. M. (1998). Time-resolved detection of surface plasmon polaritons with a scanning tunneling microscope. In Summaries of papers presented at the Conference on Lasers and Electro-Optics IEEE. https://doi.org/10.1109/CLEO.1998.676596

\section{General rights}

Copyright and moral rights for the publications made accessible in the public portal are retained by the authors and/or other copyright owners and it is a condition of accessing publications that users recognise and abide by the legal requirements associated with these rights.

- Users may download and print one copy of any publication from the public portal for the purpose of private study or research.

- You may not further distribute the material or use it for any profit-making activity or commercial gain

- You may freely distribute the URL identifying the publication in the public portal 


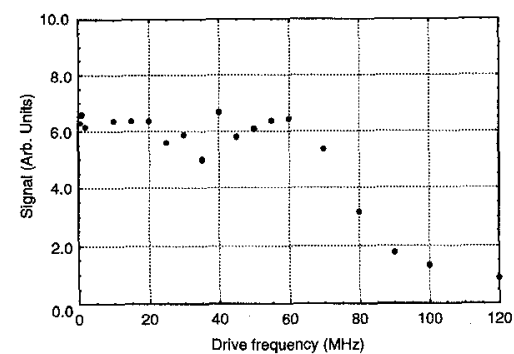

CFI7 Fig. 1. Frequency performance of the photo-induced-emf hybrid sensor.

sponse using laser diodes ( $825-935 \mathrm{~nm}$ ) as the optical source. Taken together, these two attributes (hybridized sensor, laser diode probing) can enable such a system to be economically utilized in several diverse markets. These compact packages have the potential to be configured as multipixel detector arrays with the advantages of performing single-frame inspection of complex structures or phased-array image processing for enhanced spatial resolution.

Our previous efforts have involved detectors fabricated using discrete components. In those studies, we optimized the processing of the photo-emf elements, as well as the basic electronic circuit design. In the present effort, we have fabricated compact, hybridized versions of the high-gain $(500 \mathrm{~K} \Omega)$, multiamplifier chain, using annealed Cr:GaAs elements as the active material. The T0-8 packaging reduced stray impedances, while maintaining high gain and high bandwidth, and enabled low-noise operation, with the potential for onboard thermoelectric cooling.

We measured the performance of this system using a calibrated interferometric apparatus, with an electro-optic (EO) modulator, in one of the interferometer legs (the probe), to induce a well-defined time-varying phase shift. Using this characterization facility, we evaluated the frequency dependence of the photoemf sensor, its overall sensitivity, and its noise floor. The frequency response of the sensor demonstrating an 80-Mhz bandwidth FWHM, is shown in Fig. 1.

A second concern in terms of practical receivers deals with the laser probe source. In the present investigation, we studied the response of the photo-emf detector employing laser diodes $(825-935 \mathrm{~nm})$ as the source. Successful operation using laser diodes will enable very compact systems to be realized at low cost.

For these initial experiments, we addressed the issue of photo-emf detector element response near the band edge of our GaAs active element. In this set of experiments, we employed an external-cavity SDL laser diode system, operating between 825 and $935 \mathrm{~nm}$, with an output power of up to $400 \mathrm{~mW}$ operating in a single longitudinal mode. Using a wavelength of $841 \mathrm{~nm}$, the output signal resulting from a laser-generated impulse of ultrasonic excitation in metal is shown in Fig. 2. Given these initial encouraging results, in future systems, the relatively expensive tunable externalcavity diode laser system can be replaced using a lower cost, single-frequency laser diode, such as a distributed Bragg reflector or distributed feedback.

In conclusion, we have obtained improved

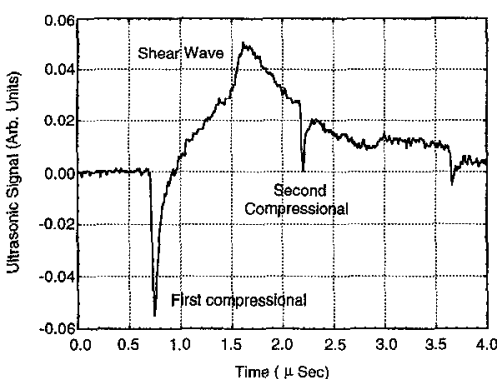

CFI7 Fig. 2. Using a diode laser probe (841 $\mathrm{nm}$ ), the photo-induced-emf hybrid sensor output signal from a laser-generated impulse of ultrasonic excitation in metal

device performance using a hybridized, monolithic photo-emf receiver, with a laser diode as a probe, with bandwidths in the range of 80 $\mathrm{MHz}$, with improved sensitivity. Using this type of photo-emf sensor, we have also theoretically examined the performance of sensor arrays.

${ }^{*}$ Melles Griot, Inc., Irvine, California

1. M.P. Petrov, S.I. Stepanov, G.S. Trofimov, Sov. Tech. Phys. Lett. 12, 379 (1986); I.A. Sokolov, S.I. Stepanov, J. Opt. Soc. Am. B 10, 1483 (1993).

2. S.I. Stepanov, Appl. Opt. 33, 915 (1994).

3. P.V. Mitchell, G.J. Dunning, T.R. O'Meara, M.B. Klein, D.M. Pepper, Rev. Prog. Quantum Nondest. Eval., Vol. 15A, D. Thompson, D. Chimenti, eds. (Plenum, New York, 1995); D.M. Pepper, G.J. Dunning, P.V. Mitchell, S.W. McCahon, M.B. Klein, T.R. O'Meara, Proc. SPIE 2703, 91 (1996).

\section{CFJ}

1:00 pm-2:30 pm Room 103

\section{Ultrafast Measurement Techniques}

Henry van Driel, University of Toronto, Presider

\section{CFJ1}

1:00 pm

Time-resolved detection of surface plasmon polaritons with a scanning tunneling microscope

Ulrich D. Keil, Taekjip Ha, ${ }^{*}$ Jacob R. Jensen, Jørn M. Hvam, Mikroelektronik Centret, DTU Bldg. 345 East, DK-2800 Lyngby, Denmark; E-mail: ulli@mic.dtu.dk

Laser-induced surface plasmon polaritons (SPPs) have been measured with a scanning tunneling microscope (STM) through heating of the tunneling junction and by rectification of the plasmon field. ${ }^{1}$ Time-resolved measurements of SPPs excited with a femtosecond laser source have been reported in reflectivity on silver. ${ }^{2}$ Here we report on the combination of these techniques. ${ }^{3}$

The SPPs are excited in an attenuated total internal reflection geometry on a 40 -nm-thick Au-layer evaporated on a prism (Fig. 1). We

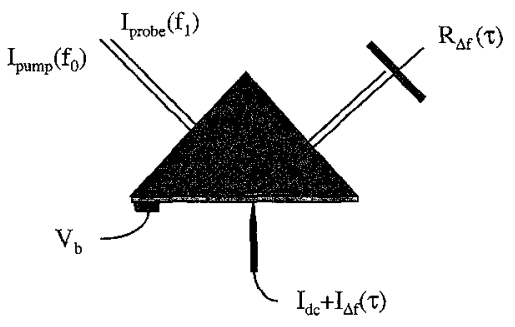

CFI1 Fig. 1. Experimental setup for timeresolved current and reflectivity measurements. Pump and probe beams are modulated at $f_{0}$ and $f_{1}$, respectively, with a difference frequency of $\Delta f$. The change in reflectivity and the change in the tunneling current are measured at $\Delta \mathrm{f}$.

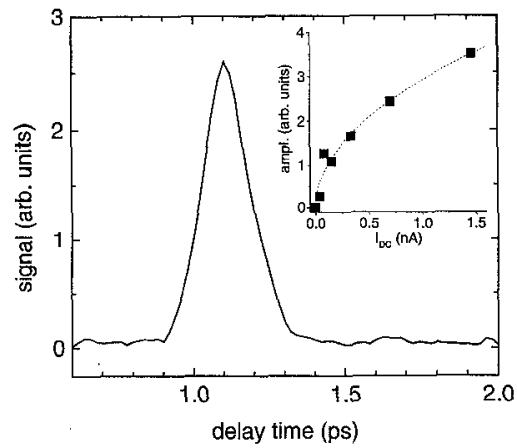

CFJ1 Fig. 2. Delay-time-dependent tunneling current with the tip under the probe beam measured at $V_{\mathrm{b}}=48 \mathrm{mV}, I_{\mathrm{dc}}=0.7 \mathrm{nA}$. The inset shows the dependence of the amplitude on $I_{\mathrm{dc}}$ for $V_{\mathrm{b}}=48 \mathrm{mV}$.

use two laser beams to excite SPPs. Pump and probe beams are $p$ polarized, do not overlap on the sample, and are modulated at fundamental frequencies $\left(f_{0}, f_{1}\right)$ of approximately $600 \mathrm{kHz}$. The laser wavelength is $840 \mathrm{~nm}$. The current (or the reflection) is measured with the lock-in locked to the difference frequency, $\Delta \mathrm{f}=1.4$ $\mathrm{kHz}$. For these high fundamental modulation frequencies, thermally induced signals disappear. The modulated tunneling current, $I_{\Delta \mathrm{f}}$ is monitored while the delay between pump and probe beams is varied. If the beams interact nonlinearly in the generation or detection mechanism, a dependence on the relative timing, $\tau$, can be measured.

Figure 2 shows the delay-time-dependent change in the tunneling current. The signal width of $200 \mathrm{fs}$ is at the resolution limit of the setup. The signal is only observed if both beams are $p$ polarized. Only in this configuration can SPPs be generated. The amplitude dependence is shown as an inset. The measurements are performed for different set points of the tunneling current in constant current mode; a higher current implies a shorter tipsample distance.

For mixing of the plasmon fields at the tunneling junction through rectification, we can only expect a delay-time-dependent contribution for a coherent superposition of the plasmon fields generated by the two laser beams. Apparently, for times longer than the laser pulse, the SPPs lose their fixed phase relation due to scattering.

The differential reflectivity measurements 


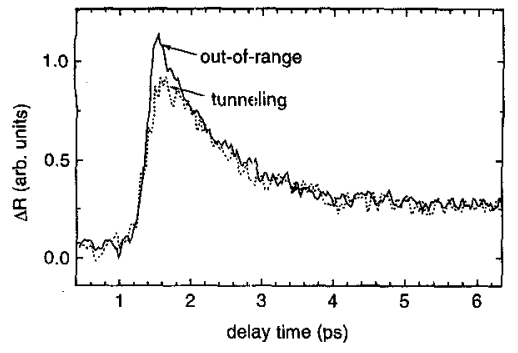

CFJ1 Fig. 3. Differential reflectivity measurement with the tip under the pump beam in and out of tunneling range.

show a much slower decay (Fig. 3). The reflectivity is, at these photon energies $(1.5 \mathrm{eV})$, proportional to the excess energy of the electron gas, and the time-resolved measurement provides a measure for the relaxation. ${ }^{2}$ The reflectivity signal is a noncoherent measurement of the plasmon decay. In tunneling range, the reflectivity is influenced by the tip during the first $300 \mathrm{fs}$.

In summary, we present the time-resolved detection of surface plasmon polaritons with an STM. The results indicate that the timeresolved signal is due to rectification of coherently superimposed plasmon voltages. The comparison with differential reflectivity measurements shows that the tip itself influences the decay of the plasmon-field coherence. Generation of the measured signal at the tunneling junction offers the possibility to observe ultrafast effects with a spatial resolution determined by the tunneling junction.

${ }^{*}$ Materials Sciences Division, Lawrence Berkeley National Laboratory, 1 Cyclotron Road, Berkeley, California 94720

1. N. Kroo et al., Europhys. Lett. 15, 289 (1991); R. Möller, et al., J. Vac. Sci. Technol. B 9, 506 (1991).

2. R.H.M. Groeneveld, R. Sprik, A, Lagendijk, Phys. Rev. B 51, 11433 (1995).

3. M.J. Feldstein, P. Vöhringer, W. Wang, N.F. Scherer, J. Phys. Chem. 100, 4739 (1996).

\section{CFJ2}

1:15 pm

Ultrafast response times and enhanced optical nonlinearity in beryllium-doped low-temperature-grown GaAs

A. Prasad, M. Haiml, I.D. Jung, J. Kunde, F. Morier-Genoud, E.R. Weber, ${ }^{\star}$ U. Siegner, U. Keller, Swiss Federal Institute of Technology Zürich, Institute of Quantum Electronics, ETH Hönggerberg HPT, CH-8093 Zürich,

Switzerland;E-mail: haiml@iqe.phys.ethz.ch

Ultrafast all-optical switching applications require materials with fast response times and large absorption modulation. For applications in laser physics, ${ }^{1}$ another key parameter is the amount of remaining absorption loss when the absorption is already saturated at high pump fluences (nonsaturable losses). In this paper, we demonstrate that beryllium doping of lowtemperature-grown (LT) GaAs considerably shortens the initial decay time of the nonlinear absorption to values of about $100 \mathrm{fs}$. Importantly, this improvement of the time response

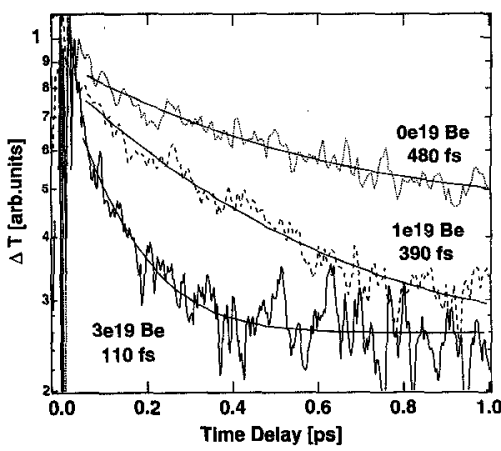

CFJ2 Fig. 1. Pump-probe signal vs time delay from bulk GaAs grown at $300^{\circ} \mathrm{C}$ : undoped sample (dotted line), Be doping concentration 1 . $10^{19} \mathrm{~cm}^{-3}$ (dashed line), Be-doping concentration $3 \cdot 10^{19} \mathrm{~cm}^{-3}$ (solid line). The data have been obtained at room temperature with 15 -fs pulses with center wavelength $750 \mathrm{~nm}$. The smooth solid lines show fits using $a+b \exp (-t / \tau)$ from which the time constant $\tau$ of the initial decay is obtained.

is accompanied by an increase of the absorption modulation and a large decrease of the nonsaturable losses. This makes Be-doped GaAs a very promising material for ultrafast all-optical switching devices.

The pump-probe scans in Fig. 1 were measured with $15-\mathrm{fs}$ pulses for above-bandgap excitation (center wavelength $750 \mathrm{~nm}$ ) on samples grown at $300^{\circ} \mathrm{C}$, either undoped or with Be-doping concentrations of $1 \cdot 10^{19}$ $\mathrm{cm}^{-3}$ or $3 \cdot 10^{19} \mathrm{~cm}^{-3}$. The initial decay time decreases from $480 \mathrm{fs}$ to $390 \mathrm{fs}$ and $110 \mathrm{fs}$ with increasing Be concentration. In undoped LT $\mathrm{GaAs}$, the initial decay of the pump-probe signal is due to trapping of band electrons into positive arsenic antisites $\mathrm{As}_{\mathrm{Ga}}{ }^{+2}$. Magnetic circular dichroism of absorption and nearinfrared absorption studies ${ }^{3}$ show that the Be acceptors have increased the $\mathrm{As}_{\mathrm{Ga}}{ }^{+}$concentration at the expense of the $\mathrm{As}_{\mathrm{Ga}}{ }^{\circ}$ concentration in the sample with $1 \cdot 10^{19} \mathrm{~cm}^{-3} \mathrm{Be}$ (see inset in Fig. 2), leading to faster trapping. However, at the highest doping level, the $\mathrm{As}_{\mathrm{Ga}}{ }^{+}$concentration is smaller than in the undoped sample, and $\mathrm{As}_{\mathrm{Ga}}{ }^{2+}$ defects or As-Be complexes ${ }^{4}$ must have formed. We conclude from the fast initial decay of the pump-probe signal in the highly doped sample that the new defect state or complex is a very efficient trap.

The strength of the absorptive nonlinearity is determined by quantitative measurements of the transmission versus the fluence of a single incident pulse (pulse width $100 \mathrm{fs}$, wavelength $830 \mathrm{~nm}$ ), from which we determine the linear transmission $\mathrm{T}_{\mathrm{o}}$, the maximum modulation $\Delta \mathrm{T}$ for pump fluences in the saturated regime, and the nonsaturable losses $T_{n s}=1-$ $\mathrm{T}_{\mathrm{s}}\left(\mathrm{T}_{\mathrm{s}}\right.$ transmission for pump fluence in the saturated regime), see Fig. 2. For undoped LT $\mathrm{GaAs}$, the modulation $\Delta \mathrm{T}$ decreases [Fig. 3(a)] and the nonsaturable losses increase [Fig. 3(b)] when the growth temperature is lowered in order to shorten the trapping time ${ }^{5}$ by an increased $\mathrm{As}_{\mathrm{Ga}}{ }^{+}$concentration. ${ }^{3}$ In contrast, the shortening of the initial decay time due to $\mathrm{Be}$ doping of LT GaAs is accompanied by a considerable increase of the modulation and a large decrease of the nonsaturable losses (Fig. $3)$.

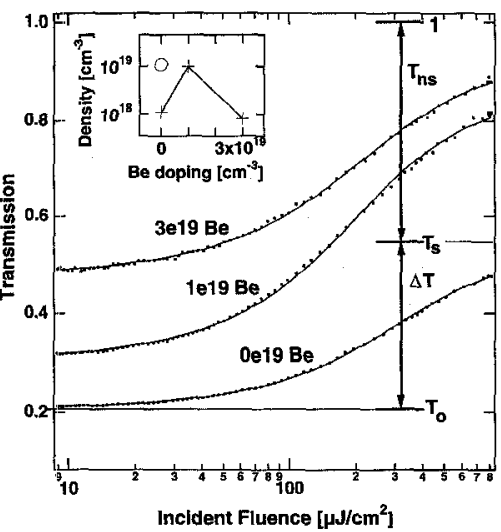

CFJ2 Fig. 2. Absolute transmission vs. fluence of a single-incident 100 -fs pulse with center wavelength $830 \mathrm{~nm}$ for different GaAs samples grown at $300^{\circ} \mathrm{C}$ (data taken at room temperature). From bottom to top: undoped sample, Bedoping concentration $1 \cdot 10^{19} \mathrm{~cm}^{-3}$, Be-doping concentration $3 \cdot 10^{19} \mathrm{~cm}^{-3}$. $\mathrm{T}_{\mathrm{o}}$ linear transmission, $T_{s}$ transmission for pump fluences in the saturated regime, $\Delta T=T_{s}-T_{o}$ modulation for pump fluences in the saturated regime, $T_{n s}=1-$ $\mathrm{T}_{\mathrm{s}}$ nonsaturable losses. Inset: defect density vs. Be-doping concentration, density of neutral arsenic antisite $\mathrm{As}_{\mathrm{Ga}^{\circ}}{ }^{\circ}$ (open circle), density of positive arsenic antisite $\mathrm{As}_{\mathrm{Ga}}{ }^{+}$(crosses). In the Bedoped samples, the density of neutral $\mathrm{As}_{\mathrm{G}_{\mathrm{a}}}{ }^{\circ}$ is below the detection threshold of about $10^{18}$ $\mathrm{cm}^{-3}$.
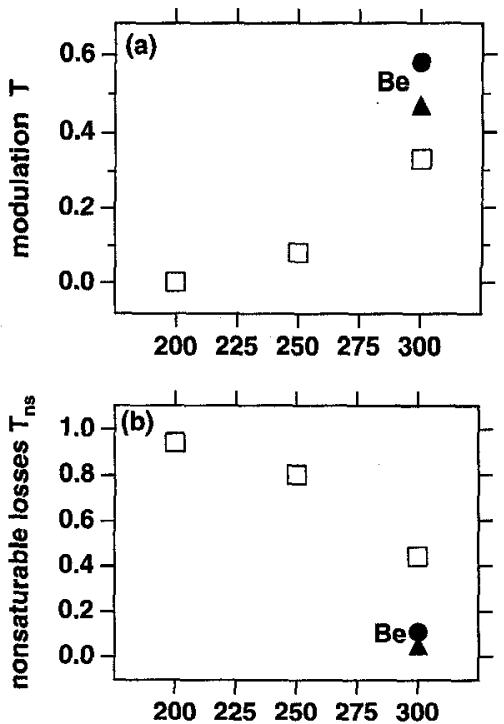

Growth Temperature $\left[{ }^{\circ} \mathrm{C}\right]$

CFJ2 Fig. 3. Modulation $\Delta \mathrm{T}$ (a) and nonsaturable losses (b) vs. growth temperature: undoped low-temperature-grown (LT) GaAs (open squares), LT GaAs with Be-doping concentration $1 \cdot 10^{19} \mathrm{~cm}^{-3}$ (filled circles), LT GaAs with Bedoping concentration $3 \cdot 10^{19} \mathrm{~cm}^{-3}$ (filled triangles).

We attribute this to the decrease of the $\mathrm{As}_{\mathrm{G}: \mathrm{a}}{ }^{\circ}$ and the increase of the ionized antisite concentration, which weakens the $\mathrm{As}_{\mathrm{Ga}}{ }^{\circ}$ to conduction band (CB) transition in Be-doped LT GaAs, demonstrated by the increase of the linear transmission (see Fig. 2). We expect that 